\title{
Fracture Bilateral Talus in Children
}

\author{
Vinit Verma, Amit Batra, Pradeep Kamboj, Suresh Bhuriya, Raj Singh, \\ Sumit Kumar, Rakesh Gupta, Narender Kumar Magu \\ Pt. B. D. Sharma PGIMS Rohtak, Rohtak, India \\ Email: verma_vinit@rediffmail.com
}

Received March 10, 2013; revised April 12, 2013; accepted April 19, 2013

Copyright (c) 2013 Vinit Verma et al. This is an open access article distributed under the Creative Commons Attribution License, which permits unrestricted use, distribution, and reproduction in any medium, provided the original work is properly cited.

\begin{abstract}
Talar body injuries are rare, particularly in children. To our knowledge, there has not been a single case report of bilateral talus fracture in a child till date. We report two cases of fracture bilateral talus in children. The first case is of a fracture separation of the distal tibial epiphysis and a fracture of the body of the talus with subluxation of ankle on right side and a fracture neck of talus on left side. The second is fracture bilateral talus with epiphyseal injury of left distal tibia. A minimal or undisplaced fracture of talus is less likely to undergo avascular necrosis than a displaced fracture but even with optimal treatment, avascular necrosis may still occur. It is of prime significance that these fractures should be diagnosed well in time to avert complications. Therefore an appropriate length of follow-up is required.
\end{abstract}

Keywords: Paediatric Talus Fracture; Bilateral; Avascular Necrosis

\section{Introduction}

In childhood and adolescence talar fractures constitute one third of all tarsal fractures. In pediatric patients, talar fractures are rare and account for only about $0.08 \%$ of all pediatric fractures [1]. Fracture of the talus is through the neck in about half of all cases. The usual mechanism of this fracture is sudden dorsiflexion on a partially plantar flexed foot that occurs due to a fall from height [2]. Skeletally immature bone is much less brittle than its adult counterpart and as a result it can sustain higher forces before fracture occurs. The talus is predominantly made up of cartilage, giving it much greater resilience than that of the adult explaining the rarity of this fracture in children [3]. Most of the fractures will be in the neck of the talus, as in the skeletally mature patients, although they are proportionally far fewer [4]. Posttraumatic complications after pediatric talus fractures have reportedly occurred more frequently after a high-energy trauma or a displaced fracture. Avascular necrosis, arthrosis, nonunion, delayed union, neurapraxia, infection and woundhealing problems account for most of the complications [5]. Talus fractures occur more commonly and with a greater severity in older children. The older the patient is, the more chances are, which shall require operative management. There is significantly less risk of osteonecrosis in younger age patients than in their old age counterparts. The outcome is more favourable in most of younger patients irrespective of the mode of treatment.
Although there is no apparent difference in the cause of the trauma leading to fractures of the talus, adolescents present with more severe fractures of the talus compared with younger children [6]. The problem with paediatric displaced talar fractures is not only incongruity of subtalar joint, but also shortening of medial column of foot which results in hindfoot varus, forefoot adduction and supination. This leads to an imbalance between the peroneal and flexor muscles [7].

We have reported two cases of fracture bilateral talus in children. The first one is a rare combination of a fracture separation of the distal tibial epiphysis and a fracture of the body of the talus with subluxation of ankle on right side and a fracture neck of talus on left side. The second is fracture bilateral talus with epiphyseal injury of left distal tibia.

\section{Case Report}

A 9 years old boy presented at night to the emergency department, with history of fall from a height of approximately 10 feet from roof of his home. He complained of intense pain in both ankles with swelling around both ankles. Radiographs of both ankles revealed a fracture of talar body with salter-Harris type IV epiphyseal separation on right side (Figures 1(a) and (b)) and fracture of the neck of talus on the left side with fracture tibia (Figures 2(a) and (b)). He was immediately given plaster of Paris back slab in neutral flexion at ankle joints with 


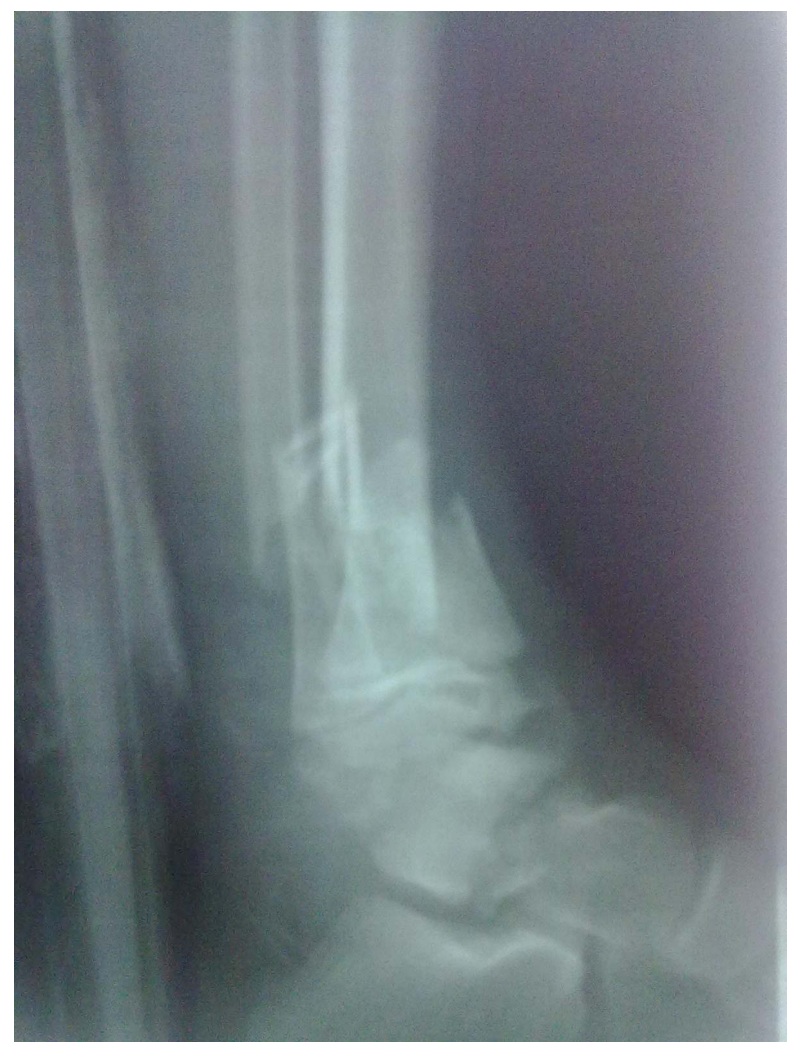

(a)

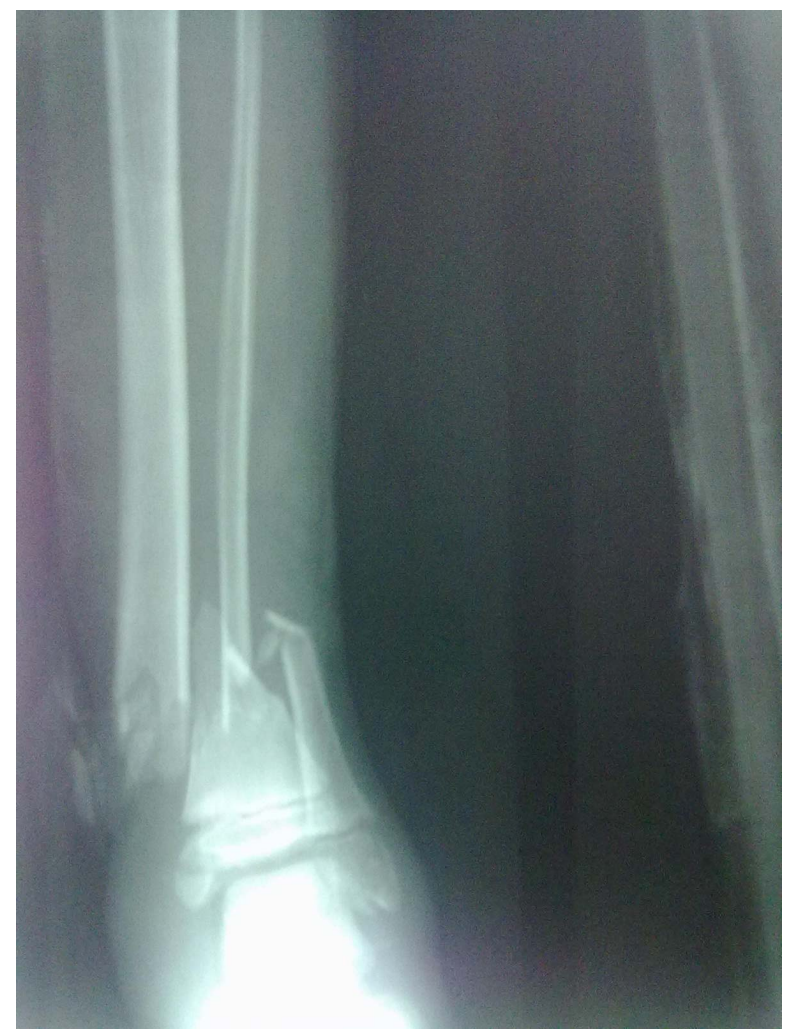

(b)

Figure 1. (a), (b) Fracture dislocation of right ankle with fracture of talus in case No. 1 (A-P and lateral views).

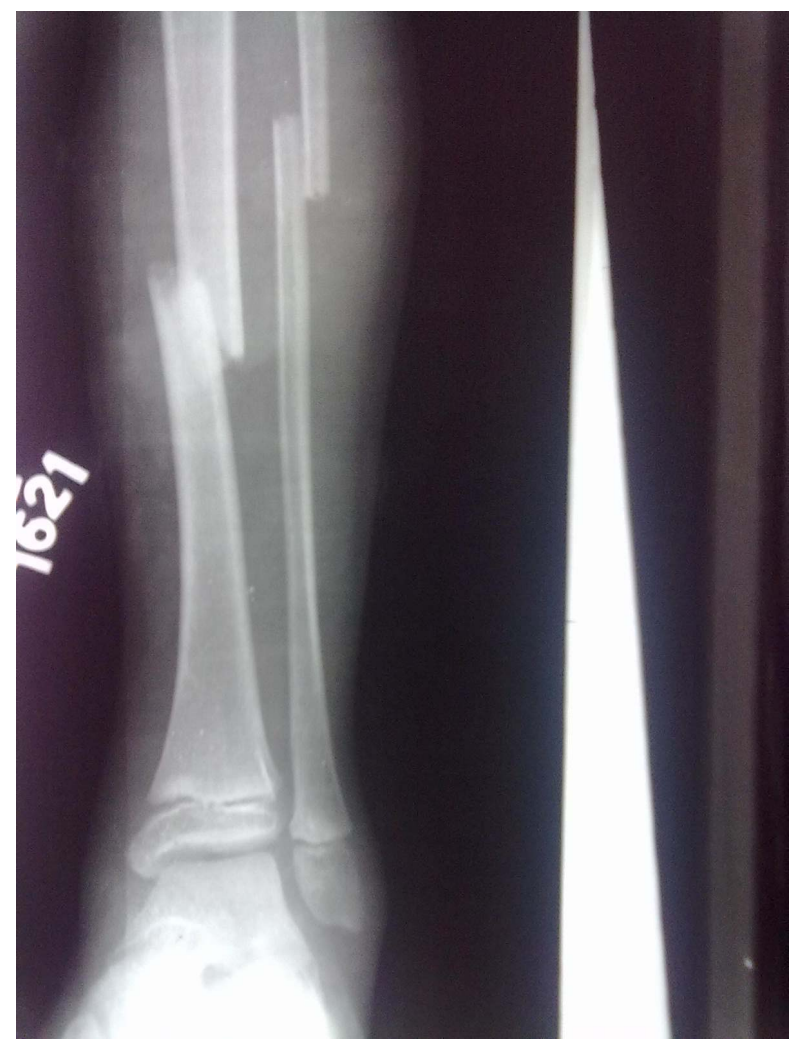

(a)

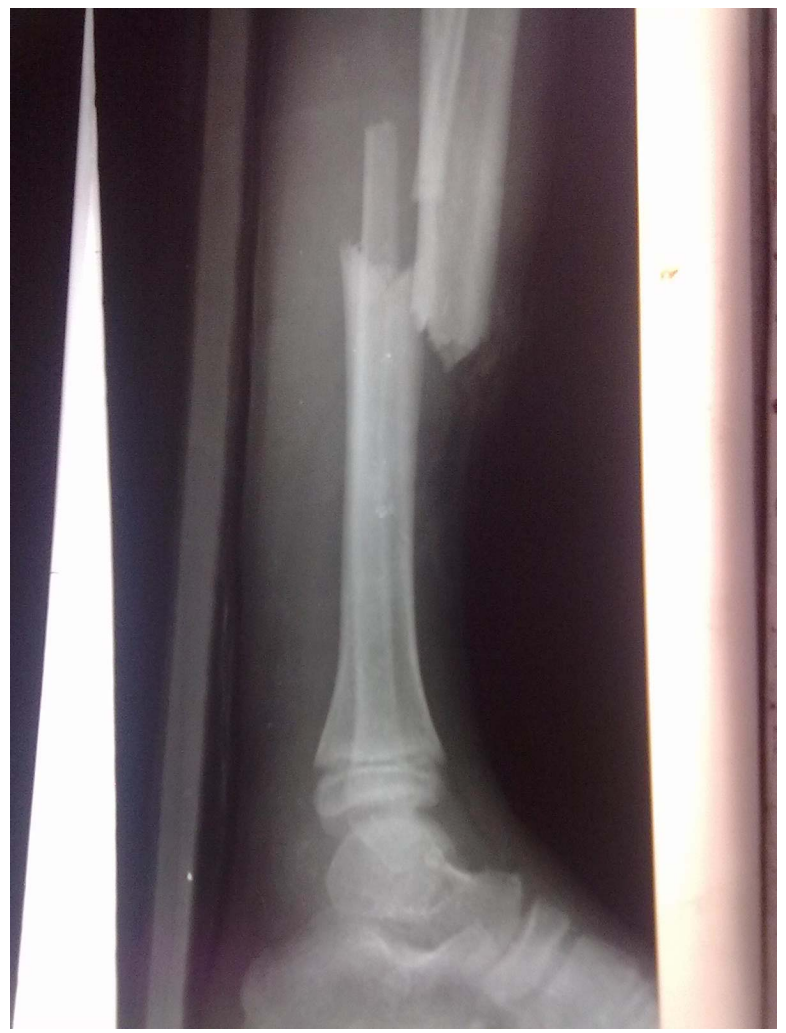

(b)

Figure 2. (a), (b) Fracture of talus and fracture of shaft of tibia (left) in case No. 1 (A-P and lateral views). 
elevation of the injured limbs and judicious ice packing. The patient was investigated and was planned for surgery on both the sides. The epiphyseal injury of distal tibia (right side) was reduced and fixed with $\mathrm{k}$ wires. Fracture of talus on right side was fixed with a lag screw and a $\mathrm{k}$ wire and supplemented by pop splint. On left side the talus fracture was fixed with two $\mathrm{k}$ wires and above knee pop cast for fracture tibia after closed reduction (Figures 3(a) and (b)).

He was kept in plaster for 8 weeks during which time he was not allowed to bear weight and had regular follow ups with radiographs at our fracture clinic. He was then taken out of plaster and physiotherapy was commenced. The patient was kept non-weight bearing for a further 2 weeks, before being allowed to weight bear. At 4 months follow-up he had no functional problems on the right side, and at his latest review 26 months post injury he practically had no problem to weight bear and had regular checkups with radiographs in our fracture clinic (Figures 4(a)-(c)).

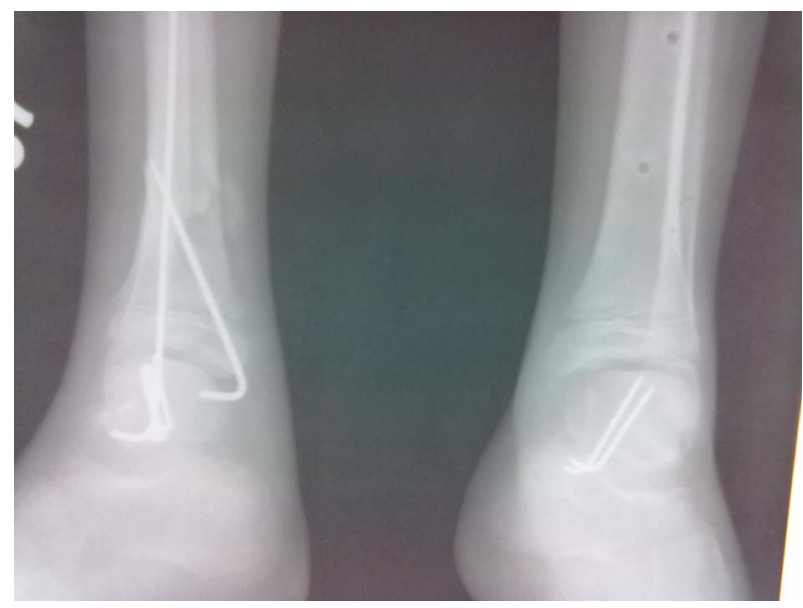

(a)

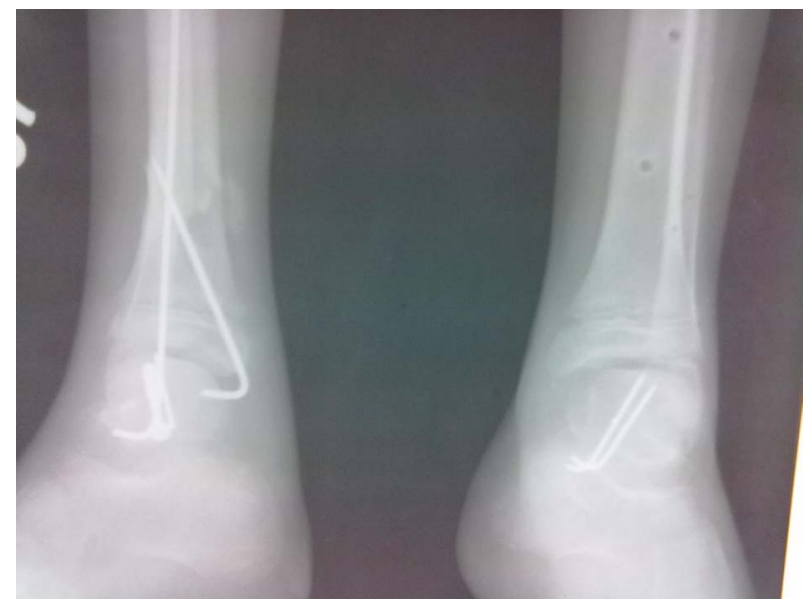

(b)

Figure 3. (a), (b) Post-operative films of right and left sides in case No. 1 .

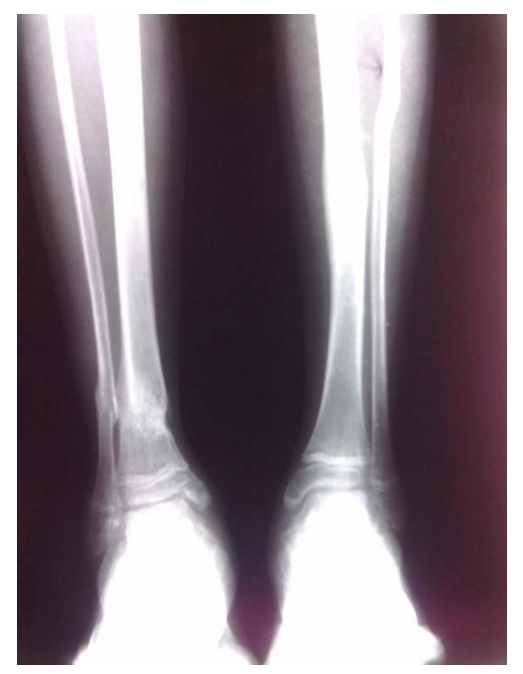

(a)

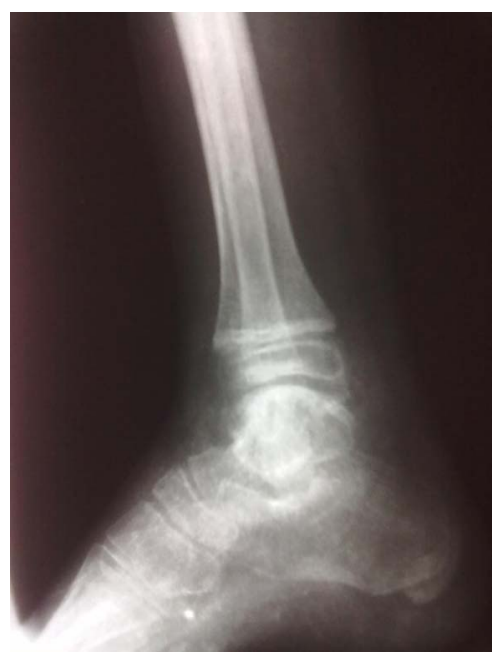

(b)

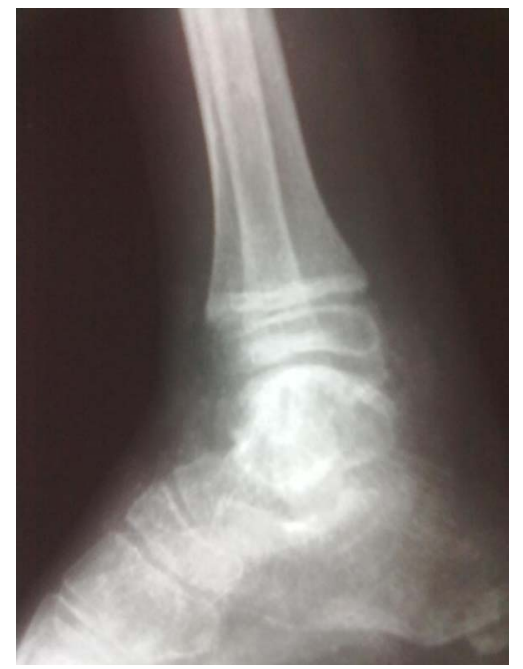

(c)

Figure 4. (a)-(c) Radiographs at final follow up. The implant has been removed and sound radiological union can be appreciated (case No. 1). 


\section{Case 2}

A 7 years old boy was admitted to casualty department with pain, swelling and inability to bear weight on both lower limbs after a road side accident. The child and his father were travelling in a bus on a seat above the rear wheels. The floor of the bus had a bump corresponding to the rear tyres of bus, on which both of them kept their feet (Figure 5). Suddenly, the bus driver applied the brakes to save a pedestrian. As the bus came to a sudden halt, the child's and his father's feet pressed violently against the bump in the floor of bus. The child complained of severe pain and inability to walk after that and was taken to hospital. The radiographs showed bilateral talus fracture with associated epiphyseal injury on left ankle. The child was subsequently operated upon on both sides with lag screws and $\mathrm{k}$ wire and a lag screw and a $\mathrm{k}$ wire for left sided epiphyseal injury (Figure 6).

His father also complained pain and swelling in foot and ankle region and was diagnosed to have lisfranc's fracture dislocation which also had to be operated. The child was kept non weight bearing and splinted for 2 months after which he was ambulated. At last follow up of 52 weeks, uneventful union had been achieved with full weight bearing (Figures 7(a) and (b)).

\section{Discussion}

Fractures of the body of the talus in the immature skeleton are rarely encountered [8]. Less common is the scenario where there is a talus fracture that is associated with an ankle fracture and dislocation in children. To our knowledge, there has not been a single case report of bilateral talus fracture in a child till date.

Talar fractures in children are rare because the talus is predominantly cartilagenous, making it much more resilient than that of the adult $[3,4]$. The authors have found only few reports of talar body fracture in children. Cases

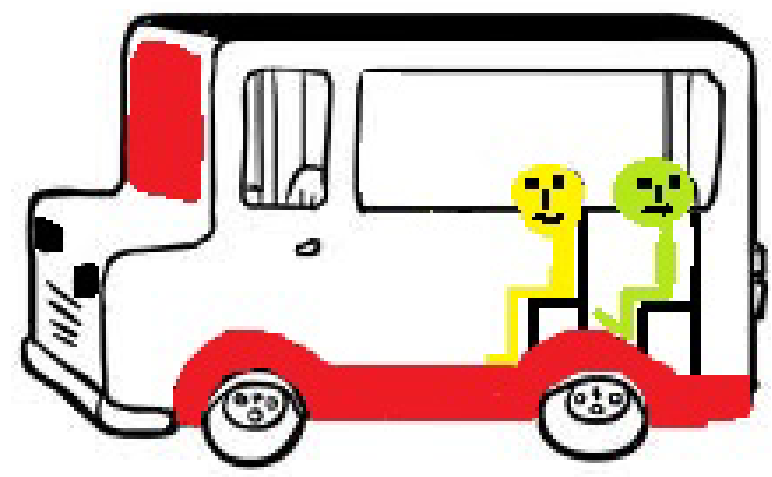

Figure 5. The pictorial representation of the mechanism of fracture in this series. The rear passenger with his feet on hump on the rear wheel of a bus suffers sudden dorsiflexion force when bus comes to a sudden stop resulting in the aforesaid fracture.

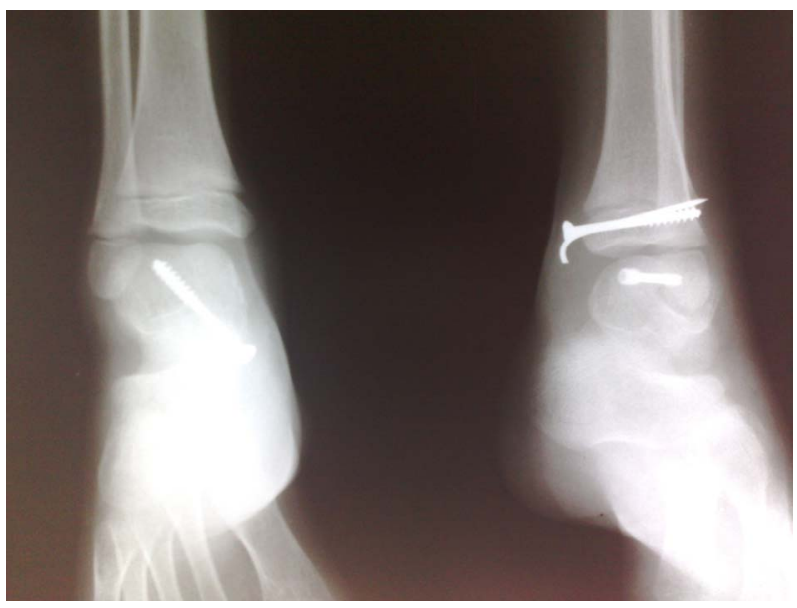

Figure 6. Post operative radiograph of case No. 2.

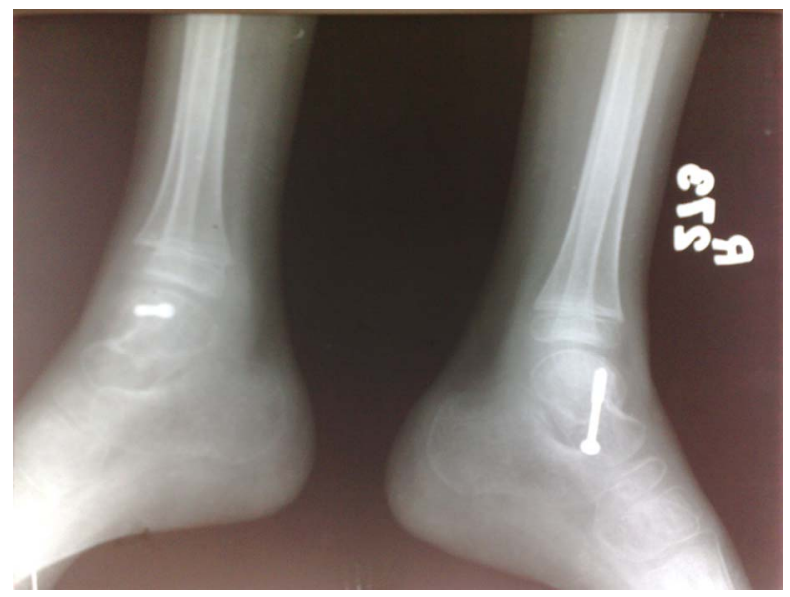

(a)

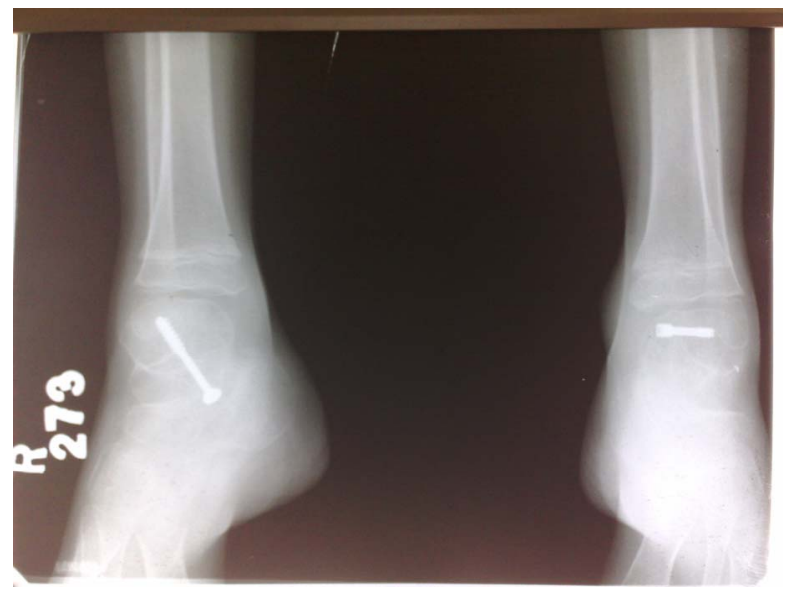

(b)

Figure 7. (a) and (b) Follow up at 2 months and 6 months respectively (case No. 2).

involving the talar body which were undisplaced have been managed conservatively in the past with excellent outcomes [9]. The concern with talar fractures in any age group is that of avascular necrosis and consequent os- 
teoarthritis. In displaced fractures of the talar body, the risk of avascular necrosis has been reported to be about $27 \%$ [10]. The published results of fractures after fixation reveal high incidence of both early and late complications, with osteonecrosis occuring in ten out of 26 cases and full talar body collapse in five out of those ten cases [11].

A major difficulty in management of these patients remains the diagnosis. Forty-seven percent of patients with chronic instability of ankle show evidence of previous talar osteochondral fracture on magnetic resonance imaging, which had not been previously diagnosed [12]. Therefore it is likely that a significant number of talar injuries may go undiagnosed. The clinical signs are variable but can include localised tenderness at the site of fracture, which can be associated with a decreased range of motion, and in more severe cases effusion and crepitus [2]. However it is important to note that there may be little or even no signs. If there is any doubt at the time of presentation as to whether there is a talar fracture further imaging is recommended. In case of late recognition of talus fractures, catastrophic results may occur in the hind-foot $[13,14]$. Hence it is prime that these fractures be diagnosed well in time to avert such complications.

Following such intra-articular injuries of ankle joint, good anatomical reduction with osteosynthesis when appropriate are mandatory even in an immature skeleton, in order to achieve a good result. Preservation of blood supply is paramount. Removal of metal work is recommended after there is evidence of healing [1]. Patient should be mobilised early without weight bearing and should remain non-weight bearing until there is evidence of sound radiological union. It is now worthwhile to suggest that looking at the mode of injury in this series our public transport buses should be modified accordingly or manufacturers should refrain from keeping the seat near rear wheels just after the bump. It is suggested that it should either be just below the seat or the area should be free of seating to avoid similar injuries.

\section{Conclusion}

Talar body injuries are uncommon, particularly in children. There exist significant differences in outcomes and complications in adult and children. These injuries can be difficult to be diagnosed and a CT scan or magnetic resonance imaging may be required. If already diagnosed, a CT scan is advised to clarify the nature of injury. A minimal or undisplaced fracture of talus is less likely to undergo avascular necrosis than a displaced fracture but even with optimal treatment, avascular necrosis may still occur. Therefore an appropriate length of follow-up is advisable. The authors advocate use of non-operative management in cases where the fracture is minimally displaced or undisplaced and in cases where there is a displacement, patient should undergo anatomical reduction and internal fixation.

\section{REFERENCES}

[1] W. E. Linhart and M. Hollwarth, "Fractures of the Talus in Children,” Unfallchirurg, Vol. 88, No. 4, 1985, pp. 168-174.

[2] D. B. Thordarson, "Talar Body Fractures,” Foot Ankle Trauma, Vol. 32, 2001, pp. 65-77.

[3] R. M. Letts and D. Gibeault, "Fractures of the Neck of the Talus in Children,” Foot Ankle Trauma, Vol. 1, No. 12, 1980, pp. 74-77.

[4] I. Spark, "Fractures of the Talus in Children," Acta Chirurgica Scandinavica, Vol. 107, No. 6, 1954, pp. 553566.

[5] J. T. Smith, T. A. Curtis, S. Spencer, J. R. Kasser, S. T. Mahan, "Complications of Talus Fractures in Children," Journal of Pediatric Orthopaedics, Vol. 30, No. 8, 2010, pp. 779-784. doi:10.1097/BPO.0b013e3181f73e6e

[6] R. Eberl, G. Singer, J. Schalamon, P. Hausbrandt and M. E. Hoellwarth, "Fractures of the Talus-Differences between Children and Adolescents,” Journal of Trauma, Vol. 68, No. 1, 2010, pp. 126-130. doi:10.1097/TA.0b013e3181a74667

[7] H. Thermann, T. Hu“fner, M. Richter, E. Schratt and H. Tscherne, "Paediatricfoot Fractures," Journal of Foot and Ankle Surgery, Vol. 7, No. 2, 2001, pp. 61-76. doi:10.1046/j.1460-9584.2001.00253.x

[8] O. Sneppen, S. Bach Christensen, O. Krogsoe and J. Lorentzen "Fracture of the Body of the Talus," Acta Orthopaedica Scandinavica, Vol. 48, No. 3, 1977, pp. 317-324. doi:10.3109/17453677708988775

[9] I. Jensen, J. U. Wester and F. Rasmussen, "Prognosis of Fracture of the Talus in Children," Acta Orthopaedica Scandinavica, Vol. 65, No. 4, 1994 pp.398-400. doi:10.3109/17453679408995478

[10] W. E. Linhart and M. Hollwarth, "Fractures of the Talus in Children,” Unfallchirurg, Vol. 88, No. 4, 1985, pp. 168-174.

[11] H. A. Vallier, S. E. Nork, S. K. Benirschke and B. J. Sangeorzan, "Surgical Treatment of talar Body Factures," The Journal of Bone \& Joint Surgery, Vol. 85-A, Suppl. 1, 2004, pp. 180-192.

[12] I. F. Anderson, K. J. Crichton, T. Grattan-Smith, et al., "Osteochondral Fractures of the Dome of the Talus," The Journal of Bone \& Joint Surgery, Vol. 71-A, No. 8, 1989, pp. 1143-1152.

[13] N. Nenopoulos, V. A. Papavasiliou and A. V. Papavasiliou, "Talus Fracture Associated with a Fracture Dislocation of the Distal Tibia in an Immature Skeleton,” Acta Orthopcedica Belgica, Vol. 69, No. 5, 2003, pp. 473-475.

[14] J. Gehr and W. Friedl, "Fracture of the Neck of the Talus in a Child,” Unfallchirurg, Vol. 109 No. 10, 2006, pp. 910-913. doi:10.1007/s00113-006-1128-z 